\title{
SELENIUM LEVELS IN PEDIATRIC PATIENTS WITH ENDOCRINE DISEASES: EVIDENCE FROM A SYSTEMATIC REVIEW
}

\author{
Vladimir Vuković1,2, Ljiljana Šaranac ${ }^{3}$, Marko Jović3 ${ }^{3}$ Bojko Bjelaković ${ }^{3}$ \\ ${ }^{1}$ Institute for Biomedicine, Eurac Research, Affiliated Institute of the University of Lübeck, Bolzano, Italy \\ ${ }^{2}$ Centre for Disease Control and Prevention, Institute of Public Health of Vojvodina, Novi Sad, Serbia \\ ${ }^{3}$ Faculty of Medicine, Niš, University of Niš, Serbia
}

\begin{abstract}
Selenium (Se) is essential micronutrient involved in several physiological processes. In many regions around the world, a suboptimal intake of Se has been reported in several health conditions, also in pediatric age. Studies on association between Se level and diseases in children reported contrasting results. We took an aim to perform a systematic review of literature and provide evidence-based conclusion on the magnitude of Se deficit in endocrine diseases in children. PubMed, ISI WoS, and Scopus databases were searched to identify eligible studies, published until July 25, 2019. Methodological quality was assessed using Newcastle-Ottawa Scale. After careful selection, 13 eligible studies were included. Majority were conducted in Turkey $(n=5)$ and Iran $(n=5)$, and sample size varied from 61 to 628 children, with a mean $( \pm S D)$ age of cases from $5.1 \pm 1.6$ months up to $13.8 \pm 4.5$ years. Eleven studies focused on different thyroid diseases, and two on children with type 1 diabetes mellitus (T1DM). In goitrous patients, Se level ranged from mean ( $\pm S D$ ), $25.71 \pm 20.68 \mu \mathrm{g} / \mathrm{L}$ to $114.9 \pm 34.1 \mu \mathrm{g} / \mathrm{L}$, while in patients with $\mathrm{T1DM}$ was $20.9 \pm 12.9 \mu \mathrm{g} / \mathrm{mL}$ and mean $(95 \% \mathrm{CI})=58.4$ $\mu g / L$ (55.0-63.09). We may conclude that goiter and thyroid dysfunction are prominent signs of Se deficiency in children. Although deficiency of iodine and selenium are usually combined in some area, our systematic review showed that Se deficiency is important goitrogenic factor in school children. Further randomized controlled trials are needed to adequately explore the role of Se in endocrine disorders in children, across different populations and regions.
\end{abstract}

Key words: Selenium, endocrine, thyroid, diabetes, pediatric, systematic review.

\section{Introduction}

Selenium $(\mathrm{Se})$ is a trace mineral and one of the essential micronutrients involved in several physiological processes [1]. It is a constituent of selenoproteins many of which are engaged in protection against oxidative stress [2], and a cofactor of many enzymes involved in several major metabolic pathways [3-6]. It is involved in thyroid metabolism as a cofactor of the glutathione peroxidase (GPx), protecting the thyroid gland against oxidative stress, and iodothyronin deiodinase enzymes, converting thyroxine (T4) to triiodothyronine (T3) [4, 6-9]. Being so important in human organism, the deficiency of Se can endanger human health and lead to misbalance of many biochemical processes further resulting in disease $[10,11]$.

Se intake and its level in the body depend on the person's diet, where major sources of Se intake are plant foods, meat and meat products, nuts (Brazil nuts), cereals, fish and shellfish $[12,13]$. In many regions around the world, people are exposed to inadequate content of $\mathrm{Se}$ in food because of the low Se content in the soils where plants and animals are grown $[6,14,15]$. Activity of Se-

Correspondence to: Vladimir Vuković M.D., Ph.D.

Centre for Disease Control and Prevention, Institute of Public Health of Vojvodina, Futoška 121, 21000 Novi Sad, Serbia

Phone: +381214897800

E-mail: vladimir.dr.vukovic@gmail.com

Received January $5^{\text {th }}, 2021 /$ Accepted January $23^{\text {rd }}, 2021$ dependent thyroid enzymes, in case of Se deficiency, could cause impairment of the thyroid metabolism even in the situation of adequate iodine intake [16]. Also, deficit of Se has been associated with the poor immune response [17, 18]. In general, association of Se deficiency with many diseases has been documented, among which the increased risk of thyroid cancer, infections and immunodepression, diabetes, Keshan's disease and endemic myxedema cretinism, mostly suggested to be due to the lack of protection against the oxidative stress $[15,19,20]$.

Suboptimal intake of Se has been reported in several health conditions also in the pediatric age [21]. In Hashimoto's thyroiditis in childhood, the deficiency of Se may promote initiation or progression of the disease $[17,22,23]$. Also, in other conditions of thyroid dysfunction in children, congenital hypothyroidism and goiter, different levels of serum Se have been demonstrated among cases and controls [16, 24]. Lower levels of Se and consequent oxidative damage are one of the possible factors involved in the etiology of diabetes mellitus in children [25]. Lower Se levels have also been reported in children with iron-deficiency anemia, dilated cardiomyopathy and acute lymphoblastic leukemia, when compared to the matched healthy controls [26, 27]. Low selenium levels also affected early neonatal morbidity in premature infants [28], and has been associated with hypoxia and respiratory diseases [29].

Studies on the association between Se level and diseases in children reported significantly lower levels of 
Se in cases or no difference between levels in cases and controls. Since endocrine health and hormonal balance are of great importance, particularly in the childhood, for normal growth and development, we took an aim to perform a comprehensive and systematic review of the literature in order to provide a general overview and draw evidencebased conclusion on the prevalence and magnitude of the Se deficit in endocrine diseases in children.

\section{Material and Methods}

\section{Literature Search Strategy}

In order to retrieve primary studies, a systematic bibliographic search was conducted in accord with the Preferred Reporting Items for Systematic Reviews and Meta-Analyses (PRISMA) guideline [30] through a multiengine search of PubMed, ISI Web of Science (WoS), and Scopus databases. For initial search of the PubMed database, the following combination of subject headings and text words was used: (((selenium OR selenate OR "selenium derivative" OR "selenium compound*" OR "antioxidant cocktail*" OR selenite OR "sodium selenite" OR selenomethionine OR selenocysteine OR ebselen OR "selenious acid" OR "selenium-binding protein*" OR "trace element*") AND (lack OR deficit OR deficiency OR reduction OR shortage OR decreas* OR insufficien*)) AND (pediatric* OR children OR child OR preschool OR school OR infan* OR adolescen* OR young*)). The remaining two databases were searched using appropriately modified PubMed query (detailed search queries are available upon request). Our search was restricted to studies conducted on humans and published up until July 25, 2019 in English, Italian or Serbian language.

\section{Study Selection}

Records from three different electronic databases were retrieved and cross-linking was performed in order to remove duplicates. Titles and abstracts of the identified records were screened and full texts of the initially eligible articles were obtained and evaluated for final eligibility. All of the steps are documented in the PRISMA flow diagram.

Studies were considered eligible if they met the following inclusion criteria: cross-sectional, case-control or cohort study design; studies on children or adolescents $\leq 18$ years old with endocrine disease; studies that evaluated serum/plasma levels of selenium. Other reviews, letters, commentaries, editorials, case studies, studies conducted on cell or animal model were further excluded. Justification for the exclusion of records was specified and any disagreement in opinion between reviewers was resolved through discussion.

\section{Data Extraction, Synthesis and Quality Assessment}

Extraction of the data from each eligible study was conducted and data was entered into standard Excel form in order to synthesize and present the results. Data on first author's name, year of study publication, country where the study was conducted, type of study design, type of endocrine disorder, total number of children, their age, percent of male participants, number of cases/controls, Se source (plasma or serum), measurement method of $\mathrm{Se}$, measured concentration of Se (in cases/controls), reported statistical findings (if available). Due to the absence of common statistical estimates of effect in the included studies, we were unable to perform a quantitative pooling of data through meta-analysis thus we used a narrative synthesis to describe the findings.

Methodological quality of the included studies was assessed using the Newcastle-Ottawa Scale (NOS) for case-controls studies [31] and the adapted version by Herzog et al. [32] for the cross-sectional studies. Three main perspectives of the methodological quality of each included study were evaluated: selection of the study groups; comparability of the groups; and the ascertainment of outcome and exposure for cross-sectional and case-control studies, respectively. This tool uses a star system to assign a maximum of nine stars for casecontrol and ten for cross-sectional studies, across the evaluated domains. Studies were classified as good $(\geq 6$ stars), moderate (3-5 stars) and low ( $<3$ stars) quality based on the maximum score. Detailed instructions for using this tool are provided elsewhere [33].

\section{Results}

\section{Literature Search and Study Selection}

A total of 4272 records were identified through the search of PubMed, ISI WoS and Scopus databases. After removing duplicates, 3312 titles and abstracts were screened, leading to a careful selection of 239 full text articles to be assessed for further eligibility. Of these, 226 articles were excluded for not meeting the inclusion criteria, leaving 13 eligible studies to be included in the qualitative synthesis [34, 35, 44-46, 36-43]. Detailed process of literature search and study selection is presented in Figure 1.

\section{Characteristics of the Studies}

Majority of the included studies were conducted in Turkey $(n=5)$ and Iran $(n=5)$, and by one in Poland, Ethiopia and Democratic Republic of the Congo (Table 1). Publication years ranged from 1990 [46] to the most recent one from 2018 [44]. Most of the studies used cross-sectional study design [34-41] while the remaining five were case-control studies [42-46]. Sample size varied from 61 [43] to 628 children [38] with mean age of cases spanning from $5.1 \pm 1.6$ months [44] to $13.8 \pm 4.5$ years [43]. Percentage of boys was different across the included studies, with a minimum of $21.95 \%$ [42] to a maximum of $71.25 \%$ [46]. Considering the investigated endocrinological disease, 11 studies focused on different thyroid disorders, namely goiter [34-41] and hypo- 
thyroidisms $[42,44]$, while the remaining two explored selenium status in children with the type 1 diabetes mellitus (T1DM) [43, 45]. Number of cases ranged from $48[35,39]$ to 280 [38] goitrous, and 35 [43] to 87 [45] children with T1DM.

Details on the selenium concentrations and main findings from the included studies are summarized in Table 2. Atomic absorption spectrometry was the most widely used method to measure the level of Se in serum [34-38, 42, 44, 46] and plasma [39-41, 43, 45]. Mean concentration of Se was different across the investigated populations and diseases. For goitrous patients, Se levels ranged from $25.71 \pm 20.68 \mu \mathrm{g} / \mathrm{L}$ [34] to $114.9 \pm 34.1 \mu \mathrm{g} / \mathrm{L}$ [36], while in patients with T1DM were $20.9 \pm 12.9 \mu \mathrm{g} / \mathrm{mL}$ [43] and $58.4 \mu \mathrm{g} / \mathrm{L}$ [45].

In general, level of Se was lower in children with goiter [35, 36, 39, 41] and in children with T1DM [43] or there was no difference reported between cases and controls in children with goiter and Hashimoto thyroiditis $[38,42]$. Studies that had investigated correlation of Se level and several clinical parameters reported different findings, negative [34, 41], positive [39, 42], mixed
$[38,44]$ some positive and some negative, and no significant correlation $[35,37,40]$ in thyroid patients. In particular, a study by Gashu and colleagues reported that serum Se was negatively associated with T4, but positively with T3 concentration, and that a unit increase in concentration of Se could increase the serum T3 concentration by a factor of 0.16 [38]. On the other side, Cinaz et al. demonstrated the lack of correlation between serum Se levels and thyroid function tests in their study [35]. In two studies conducted on diabetic patients, a negative correlation was reported between Se level and glycosylated hemoglobin (HbA1c) level [43], while Salmonowicz et al. reported non-significant correlations across different parameters [45]. Overall, the body of evidence in our review was characterized by a moderate to high quality level. Methodological quality of the included studies was good, especially of those cross-sectional study design, where two studies [34, 38] scored maximum stars across all evaluated domains. Case-control studies were mostly of moderate to good methodological quality (Table 3 ).

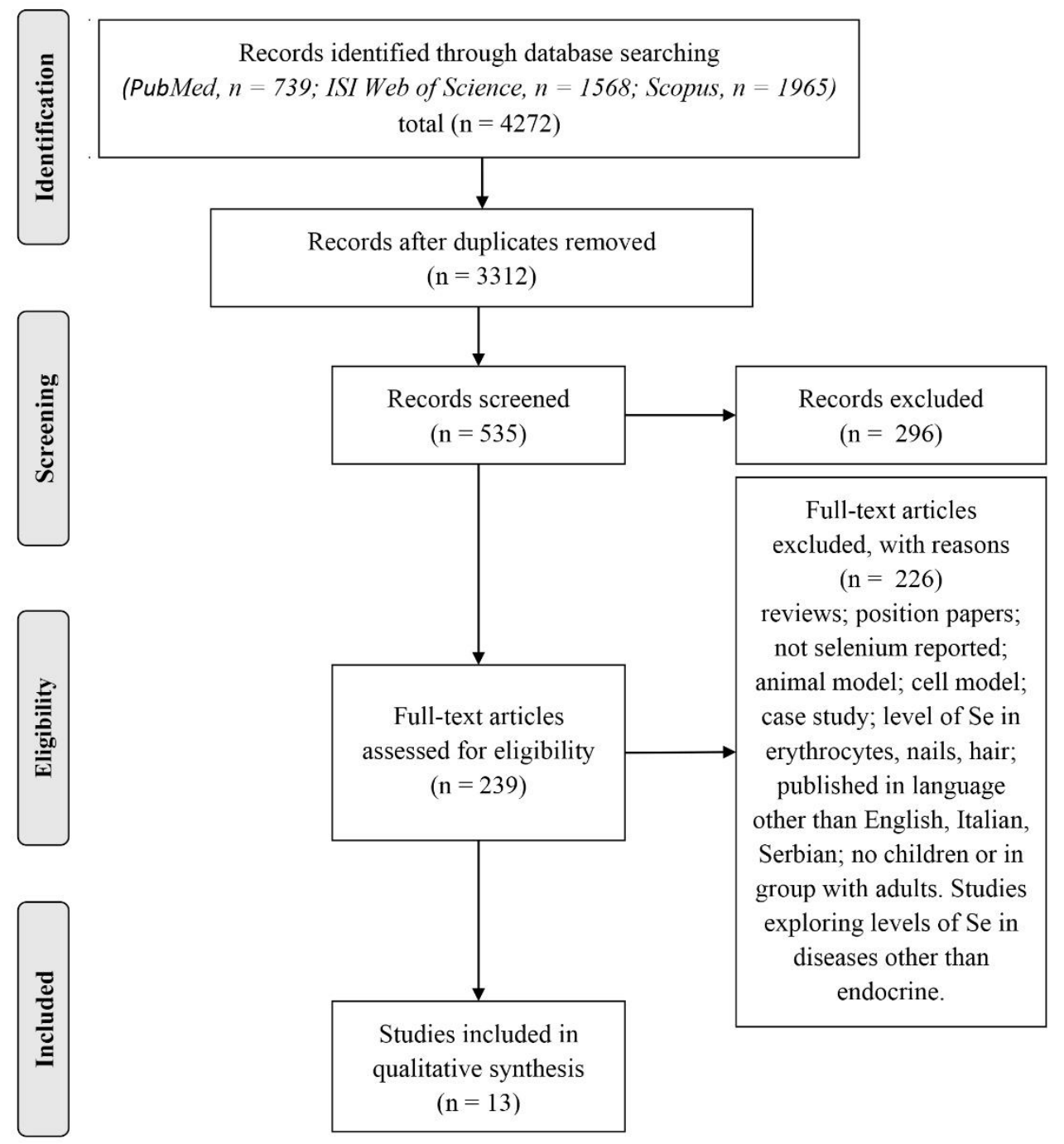

Fig. 1 Flowchart depicting literature search and study selection process 
Table 1 General characteristics of the included studies

\begin{tabular}{|c|c|c|c|c|c|c|c|}
\hline First author, year & Country & Study design & $\begin{array}{l}\text { Endocrine } \\
\text { disease }\end{array}$ & $\begin{array}{l}\text { Total } \\
\text { number of } \\
\text { children }\end{array}$ & $\begin{array}{l}\text { Age range, mean } \pm \mathrm{SD} \\
\text { (years) }\end{array}$ & $\begin{array}{l}\text { Males, } \\
\text { n }(\%)\end{array}$ & $\begin{array}{l}\text { Number }(\mathrm{n}) \text { of cases } \\
\text { and controls }\end{array}$ \\
\hline Aydin, 2002 [34] & Turkey & $\begin{array}{l}\text { Cross- } \\
\text { sectional }\end{array}$ & Goiter & 73 & $\begin{array}{c}\text { Range }=7-12 \\
\text { mean } \pm \mathrm{SD}= \\
9.56 \pm 1.77\end{array}$ & $\begin{array}{c}35 \\
(48 \%)\end{array}$ & $\begin{array}{l}\text { Cases (56); } \\
\text { controls (17) }\end{array}$ \\
\hline Cinaz, 2004 [35] & Turkey & $\begin{array}{l}\text { Cross- } \\
\text { sectional }\end{array}$ & Goiter & 165 & $\begin{array}{c}\text { Mean } \pm \text { SD }= \\
9.04 \pm 1.91\end{array}$ & NR & $\begin{array}{c}\text { Cases (48); } \\
\text { controls (117) }\end{array}$ \\
\hline $\begin{array}{l}\text { Dabbaghmanesh, } \\
2007 \text { [36] }\end{array}$ & Iran & $\begin{array}{l}\text { Cross- } \\
\text { sectional }\end{array}$ & Goiter & 500 & Range $=8-13$ & NR & $\begin{array}{c}\text { Cases (198); controls } \\
\text { (302) }\end{array}$ \\
\hline $\begin{array}{l}\text { Erdogan, } 2001 \\
{[37]}\end{array}$ & Turkey & $\begin{array}{l}\text { Cross- } \\
\text { sectional }\end{array}$ & $\begin{array}{c}\text { Goiter } \\
\text { (endemic) }\end{array}$ & 251 & Range $=9-11$ & $\begin{array}{c}123 \\
(49.01 \%)\end{array}$ & $\begin{array}{l}\text { Chidren from cities: } \\
\text { Ankara (62), } \\
\text { Kastamonu (64), } \\
\text { Bayburt (59), and } \\
\text { Kastamonu (66) }\end{array}$ \\
\hline Gashu, 2016 [38] & Ethiopia & $\begin{array}{l}\text { Cross- } \\
\text { sectional }\end{array}$ & Goiter & 628 & $\begin{array}{c}\text { Range }=4.5-5 \\
\text { mean } \pm S D= \\
4.74 \pm 0.15\end{array}$ & $\begin{array}{c}311 \\
(49.52 \%)\end{array}$ & $\begin{array}{c}\text { Cases }(280) ; \\
\text { controls }(348)\end{array}$ \\
\hline Giray, 2001 [39] & Turkey & $\begin{array}{l}\text { Cross- } \\
\text { sectional }\end{array}$ & Goiter & 121 & Range $=15-18$ & $\begin{array}{c}52 \\
(42.98 \%)\end{array}$ & $\begin{array}{l}\text { Cases (48); controls in- } \\
\text { region controls (49), } \\
\text { and out-region (24) }\end{array}$ \\
\hline $\begin{array}{l}\text { Hashemipour, } \\
2008 \text { [40] }\end{array}$ & Iran & $\begin{array}{l}\text { Cross- } \\
\text { sectional }\end{array}$ & Goiter & 219 & $\begin{aligned} \text { Range } & =7-13 ; \\
\text { mean } \pm S D & =9.3 \pm 1.0\end{aligned}$ & $\begin{array}{c}101 \\
(46.12 \%)\end{array}$ & $\begin{array}{l}\text { Cases }(108) ; \\
\text { controls }(111)\end{array}$ \\
\hline $\begin{array}{l}\text { Keshteli, } 2009 \\
\text { [41] }\end{array}$ & Iran & $\begin{array}{l}\text { Cross- } \\
\text { sectional }\end{array}$ & Goiter & 168 & $\begin{array}{c}\text { Range }=6-13 ; \\
\text { mean } \pm \mathrm{SD}= \\
9.39 \pm 1.18 \text { (girls) and } \\
9.47 \pm 1.12 \text { (boys) }\end{array}$ & $\begin{array}{c}71 \\
(42.26 \%)\end{array}$ & $\begin{array}{l}\text { Case (96); } \\
\text { controls (72) }\end{array}$ \\
\hline $\begin{array}{l}\text { Nourbakhsh, } 2015 \\
\text { [42] }\end{array}$ & Iran & Cross-control & $\begin{array}{l}\text { Hashimoto's } \\
\text { thyroiditis and } \\
\text { hypothyroidism }\end{array}$ & 82 & $\begin{array}{c}\text { Children with } \\
\text { Hashimoto's } \\
\text { thyroiditis, } \\
\text { mean } \pm \mathrm{SD}=13.0 \pm 4.2 ; \\
\text { with hypothyroidism, } \\
\text { mean } \pm \mathrm{SD}=11.4 \pm 3.3 \text {; } \\
\text { controls, mean } \pm \mathrm{SD}= \\
12.4 \pm 3.0\end{array}$ & $\begin{array}{c}18 \\
(21.95 \%)\end{array}$ & $\begin{array}{l}\text { Cases: Hashimoto's } \\
\text { thyroiditis (35), } \\
\text { hypothyroidism (22); } \\
\text { and controls (30) }\end{array}$ \\
\hline Ozenc, 2015 [43] & Turkey & Cross-control & $\begin{array}{l}\text { Type } 1 \text { diabetes } \\
\text { mellitus } \\
\text { (T1DM) }\end{array}$ & 61 & $\begin{array}{c}\text { Cases, } \\
\text { mean } \pm \mathrm{SD}=13.8 \pm 4.5 ; \\
\text { controls, mean } \pm \mathrm{SD}= \\
12.8 \pm 3.3\end{array}$ & $\begin{array}{c}34 \\
(55,74 \%)\end{array}$ & $\begin{array}{l}\text { Cases }(35) ; \\
\text { controls }(26)\end{array}$ \\
\hline $\begin{array}{l}\text { Rostami, } 2018 \\
\text { [44] }\end{array}$ & Iran & Cross-control & $\begin{array}{c}\text { Congenital } \\
\text { hypothyroidism }\end{array}$ & 99 & $\begin{array}{c}\text { Cases, range }=0.25-1, \\
\text { mean } \pm \mathrm{SD}= \\
0.42 \pm 0.13 \\
\text { controls, range }= \\
0.08-1.25 \\
\text { mean } \pm \mathrm{SD}= \\
0.52 \pm 0.12\end{array}$ & $\begin{array}{c}49 \\
(49,50 \%)\end{array}$ & $\begin{array}{l}\text { Cases (39); } \\
\text { controls (60) }\end{array}$ \\
\hline $\begin{array}{l}\text { Salmonowicz, } \\
2014 \text { [45] }\end{array}$ & Poland & Cross-control & $\begin{array}{l}\text { Type } 1 \text { diabetes } \\
\text { mellitus } \\
\text { (T1DM) }\end{array}$ & 155 & $\begin{array}{l}\text { Children with T1DM } \\
\text { range }=2-19, \\
\text { mean } \pm \mathrm{SD}=13.0 \pm 4.0 ; \\
\text { siblings range }= \\
4.5-16.5, \text { mean } \pm \mathrm{SD}= \\
13.2 \pm 3.7 ; \text { and } \\
\text { control groups } \\
\text { range }=10.5-18, \\
\text { mean } \pm \mathrm{SD}=14.8 \pm 2.2\end{array}$ & $\begin{array}{c}79 \\
(50,97 \%)\end{array}$ & $\begin{array}{l}\text { Cases (87); } \\
\text { controls: siblings of the } \\
\text { patients with T1DM } \\
\text { (27 children) and } \\
\text { healthy (41) children }\end{array}$ \\
\hline $\begin{array}{l}\text { Vanderpas, } 1990 \\
\text { [46] }\end{array}$ & $\begin{array}{l}\text { Republic of } \\
\text { Zaire } \\
\text { (Democratic } \\
\text { Republic of } \\
\text { the Congo) }\end{array}$ & Cross-control & $\begin{array}{l}\text { Endemic } \\
\text { myxedematous } \\
\text { cretinism }\end{array}$ & 80 & $\begin{array}{l}\text { Cases, range }=3-25 ; \\
\text { controls, range }=9-18\end{array}$ & $\begin{array}{c}57 \\
(71,25 \%)\end{array}$ & $\begin{array}{l}\text { Cases (28); } \\
\text { controls (52) }\end{array}$ \\
\hline
\end{tabular}


Table 2 Selenium concentrations and main findings from the included studies

\begin{tabular}{|c|c|c|c|c|}
\hline First author, year & $\begin{array}{l}\text { Biological } \\
\text { sample for } \\
\text { selenium } \\
\text { measurement }\end{array}$ & $\begin{array}{l}\text { Measurement method } \\
\text { for selenium } \\
\text { concentration }\end{array}$ & $\begin{array}{l}\text { Selenium concentrations in cases } \\
\text { and controls }\end{array}$ & Reported correlations \\
\hline Aydin, 2002 [34] & Serum & $\begin{array}{l}\text { Atomic absorption } \\
\text { spectrometry }\end{array}$ & $\begin{array}{c}\text { Goitrous, mean } \pm \mathrm{SD}= \\
25.71 \pm 20.68 \mu \mathrm{g} / \mathrm{L}) ; \text { non-goitrous, } \\
\text { mean } \pm \mathrm{SD}=47.76 \pm 22.84 \mu \mathrm{g} / \mathrm{L}\end{array}$ & $\begin{array}{l}\text { Negative correlation was reported between } \\
\text { thyroid volume and Se level } \\
(\mathrm{r}=0.32, \rho<0.05) \text {. }\end{array}$ \\
\hline Cinaz, 2004 [35] & Serum & $\begin{array}{l}\text { Spectrophotometer } \\
\text { (Unicam 939 AA) }\end{array}$ & $\begin{array}{c}\text { Goitrous, mean } \pm \mathrm{SD}=52.39 \pm \\
10.87 \mathrm{ng} / \mathrm{mL} ; \text { non-goitrous group, } \\
\text { mean } \pm \mathrm{SD}=58.94 \pm 15.42 \mathrm{ng} / \mathrm{mL} ; \\
\mathrm{p}=0.002\end{array}$ & $\begin{array}{l}\text { Serum Se levels did not correlate with the } \\
\text { thyroid function tests, and T3, T4, and } \\
\text { TSH levels are found to be similar in } \\
\text { goitrous and nongoitrous children. }\end{array}$ \\
\hline $\begin{array}{l}\text { Dabbaghmanesh, } \\
2007 \text { [36] }\end{array}$ & Serum & $\begin{array}{l}\text { Atomic absorption } \\
\text { spectrometry } \\
\text { (Chemtech Anaytical } \\
\text { CTA 2000, AAS) }\end{array}$ & $\begin{array}{c}\text { Goiterous, mean } \pm \mathrm{SD}= \\
114.9 \pm 34.1 \mu \mathrm{g} / \mathrm{L} ; \text { non-goitrous, } \\
\text { mean } \pm \mathrm{SD}=121.9 \pm 28.7 \mu \mathrm{g} / \mathrm{L} \\
\mathrm{p}<0.05\end{array}$ & C \\
\hline $\begin{array}{l}\text { Erdogan, } 2001 \\
{[37]}\end{array}$ & Serum & $\begin{array}{l}\text { Atomic absorption } \\
\text { spectrometer (Hitachi } \\
\text { Z-8200@ polarized } \\
\text { Zeeman) }\end{array}$ & $\begin{array}{c}\text { Study area: Ankara, mean } \pm \mathrm{SD}= \\
54.82 \pm 15.73 \mathrm{ng} / \mathrm{mL} ; \text { Kastamonu, } \\
\text { mean } \pm \mathrm{SD}=50.99 \pm 13.38 \mathrm{ng} / \mathrm{mL} ; \\
\text { Bayburt, mean } \pm \mathrm{SD}= \\
57.54 \pm 14.60 \mathrm{ng} / \mathrm{mL} ; \text { and Trabzon, } \\
\text { mean } \pm \mathrm{SD}=52.22 \pm 14.30 \mathrm{ng} / \mathrm{mL}\end{array}$ & $\begin{array}{l}\text { No significant correlations between serum } \\
\text { Se concentrations and studied parameters } \\
\text { (i.e., Thyroid volume, thiocyanate (SCN-) } \\
\text { overload, thyroid hormones, sensitive TSH } \\
\text { (sTSH) levels, and urinary iodine } \\
\text { concentrations (UICs)) was detected. }\end{array}$ \\
\hline Gashu, 2016 [38] & Serum & $\begin{array}{l}\text { Mass spectrometer } \\
\text { (PerkinElmer, } \\
\text { ELAN9000, Norwalk, } \\
\text { CT, USA). }\end{array}$ & $\begin{array}{l}\mathrm{NR} \text { (mean Se concentrations of } \\
\text { goitrous and non-goitrous children } \\
\text { were not significantly different, } \\
\mathrm{p}>0.05 \text { ). Entire study population, } \\
\text { median }=61.4 \mu \mathrm{g} / \mathrm{L} \\
\text { range }=10.7-290.9 \mu \mathrm{g} / \mathrm{L}\end{array}$ & $\begin{array}{l}\text { Serum Se was negatively correlated to T4 } \\
\text { concentration }(r=-0.22, p<0.01) \text {. }\end{array}$ \\
\hline Giray, 2001 [39] & Plasma & $\begin{array}{l}\text { Spectrofluorometric } \\
\text { method }\end{array}$ & $\begin{array}{c}\text { Total goiter group, mean } \pm \mathrm{SD}= \\
67.1 \pm 10.9 \mu \mathrm{g} / \mathrm{L} ; \text { total in-region } \\
\text { control, mean } \pm \mathrm{SD}=75.0 \pm 14.6 \mu \mathrm{g} / \mathrm{L} ; \\
\text { and out-region control group, } \\
\text { mean } \pm \mathrm{SD}=75.3 \pm 12.8 \mu \mathrm{g} / \mathrm{L}\end{array}$ & $\begin{array}{l}\text { In "all severely deficient children" a } \\
\text { positive correlations of urinary iodine and } \\
\text { Se were observed }(r=0.42, p<0.05) \text {. }\end{array}$ \\
\hline $\begin{array}{l}\text { Hashemipour, } \\
2008 \text { [40] }\end{array}$ & Plasma & $\begin{array}{l}\text { Atomic absorption } \\
\text { spectrometer }\end{array}$ & $\begin{array}{c}\text { Goitrous, mean } \pm \mathrm{SD}=62.7 \mathrm{~g} / \mathrm{L} ; \text { non- } \\
\text { goitrous, mean } \pm \mathrm{SD}=60.8 \mathrm{~g} / \mathrm{L} ; \mathrm{p}= \\
0.42\end{array}$ & $\begin{array}{c}\text { No correlation was found between serum } \\
\text { Se concentration and baseline data of the } \\
\text { patients including height, weight, BMI and } \\
\text { age. }\end{array}$ \\
\hline $\begin{array}{l}\text { Keshteli, } 2009 \\
\text { [41] }\end{array}$ & Plasma & $\begin{array}{l}\text { Atomic absorption } \\
\text { spectrometer }\end{array}$ & $\begin{array}{c}\text { Goitrous, mean } \pm \mathrm{SD}= \\
66.86 \pm 21.82 \mu \mathrm{g} / \mathrm{L} ; \text { non-goitrous } \\
\text { children, mean } \pm \mathrm{SD}= \\
76.67 \pm 23.33 \mu \mathrm{g} / \mathrm{L} ; \mathrm{p}=0.006\end{array}$ & $\begin{array}{l}\text { In goitrous children, Se level was reversely } \\
\text { correlated with age }(\mathrm{r}=-0.24, \mathrm{p}=0.02), \\
\text { BMI }(\mathrm{r}=-0.20, \mathrm{p}=0.05) \text { and body surface } \\
\text { area }(\mathrm{r}=-0.26, \mathrm{p}=0.01) \text { and was positively } \\
\text { correlated with } \mathrm{T} 4(\mathrm{r}=0.22, \mathrm{p}=0.03) .\end{array}$ \\
\hline $\begin{array}{l}\text { Nourbakhsh, } \\
2015 \text { [42] }\end{array}$ & Serum & $\begin{array}{l}\text { Atomic absorption } \\
\text { spectrometer (Con- } \\
\text { trAA 700, Analytik } \\
\text { Jena AG, Jena, } \\
\text { Germany) }\end{array}$ & $\begin{array}{c}\text { Hashimoto's thyroiditis, mean } \pm \mathrm{SD}= \\
91.6 \pm 17.7 \mu \mathrm{g} / \mathrm{L} ; \text { hypothyroidism, } \\
\text { mean } \pm \mathrm{SD}=85.9 \pm 14.8 \mu \mathrm{g} / \mathrm{L} ; \\
\text { controls, mean } \pm \mathrm{SD}=97.2 \pm 29.4 \mu \mathrm{g} / \mathrm{L}\end{array}$ & $\begin{array}{l}\text { Se levels did not show any significant } \\
\text { correlation with glutathione peroxidase but } \\
\text { it had a significant correlation with } \\
\text { selenoprotein } \mathrm{P}(\mathrm{r}=0.34, \mathrm{p}=0.02)\end{array}$ \\
\hline Ozenc, 2015 [43] & Plasma & $\begin{array}{l}\text { Atomic absorption } \\
\text { spectrophotometry } \\
\text { (Varian Techtron Pty. } \\
\text { Ltd., Victoria, } \\
\text { Australia) }\end{array}$ & $\begin{array}{c}\mathrm{T} 1 \mathrm{DM}, \text { mean } \pm \mathrm{SD}= \\
20.9 \pm 12.9 \mu \mathrm{g} / \mathrm{mL} ; \text { controls, } \\
\text { mean } \pm \mathrm{SD}=32.6 \pm 10.2 \mu \mathrm{g} / \mathrm{mL}\end{array}$ & $\begin{array}{l}\text { There was a negative correlation between } \\
\text { Se and HbA1c levels } \\
(\mathrm{r}=-0.44, \mathrm{p}<0.01)\end{array}$ \\
\hline $\begin{array}{l}\text { Rostami, } 2018 \\
{[44]}\end{array}$ & Serum & $\begin{array}{l}\text { Atomic absorption } \\
\text { spectroscopy }\end{array}$ & $\begin{array}{l}\text { Hypothyroidism, mean } \pm \mathrm{SD}= \\
10.5 \pm 0.65 \mathrm{mg} / \mathrm{L} ; \text { controls, } \\
\text { mean } \pm \mathrm{SD}=55.17 \pm 8.55 \mathrm{mg} / \mathrm{L} \\
\mathrm{p}=0.01\end{array}$ & $\begin{array}{l}\text { No statistically significant correlations } \\
\text { were found between Se concentration and } \\
\text { TSH level }(\mathrm{r}=0.13, \mathrm{p}=0.29) \text {, and FT4 } \\
\text { concentration }(\mathrm{r}=0.07, \mathrm{p}=0.54 \text { ). There } \\
\text { was a significant correlation between } \\
\text { prooxidant-antioxidant balance (PAB) } \\
\text { value and serum Se concentration, and also } \\
\text { between Se concentration and MCV (for } \\
\text { all parameters, } \mathrm{p}>0.05 \text { ) }\end{array}$ \\
\hline $\begin{array}{l}\text { Salmonowicz, } \\
2014 \text { [45] }\end{array}$ & Plasma & $\begin{array}{l}\text { Atomic absorption } \\
\text { spectrophotometer } \\
\text { (SOLAAR M6, } \\
\text { Thermo Elemental, } \\
\text { Great Britain) }\end{array}$ & $\begin{array}{c}\text { Patients with T1DM, mean } \\
(95 \% \mathrm{CI})=58.4 \mu \mathrm{g} / \mathrm{L}(55.0-63.09) \\
\text { siblings of patients with T1DM, } \\
\text { mean, }(95 \% \mathrm{CI})=53.45 \mu \mathrm{g} / \mathrm{L}(46.09- \\
65.21) ; \text { healthy controls, mean } \\
(95 \% \mathrm{CI})=53.3 \mu \mathrm{g} / \mathrm{L}(45.78-70.17)\end{array}$ & $\begin{array}{l}\text { In patients with T1DM no significant } \\
\text { correlations were found between Se and: } \\
\mathrm{HbA1c}(\mathrm{r}=0.2, \mathrm{p}=0.07), \text { age }(\mathrm{r}=0.01 \\
\mathrm{p}=0.89), \mathrm{TC}(\mathrm{r}=-0.05, \mathrm{p}=0.72), \mathrm{TG} \\
(\mathrm{r}=-0.07, \mathrm{p}=0.59), \mathrm{HDL}-\mathrm{C}(\mathrm{r}=-0.12 \\
\mathrm{p}=0.45), \mathrm{LDL}-\mathrm{C}(\mathrm{r}=-0.02, \mathrm{p}=0.89) \\
\text { TC/HDL-C }(\mathrm{r}=0.20, \mathrm{p}=0.22)\end{array}$ \\
\hline $\begin{array}{l}\text { Vanderpas, } 1990 \\
\text { [46] }\end{array}$ & Serum & Spectrofluorimetry & $\begin{array}{c}\text { Cases, mean } \pm \mathrm{SD}= \\
443 \pm 188 \mathrm{nmol} / \mathrm{L} ; \text { controls, } \\
\text { mean } \pm \mathrm{SD}=343 \pm 176 \mathrm{nmol} / \mathrm{L} ; \mathrm{p}>0.1\end{array}$ & NR \\
\hline
\end{tabular}

$\mathrm{NR}=$ not reported; $\mathrm{SD}=$ standard deviation; $\mathrm{T} 1 \mathrm{DM}=$ Type 1 diabetes mellitus 
Table 3 Methodological quality of the included studies

\begin{tabular}{|c|c|c|c|c|}
\hline First author, year & Study design & Selection & Comparability & Outcome/Exposure \\
\hline Aydin, 2002 [34] & $\mathrm{CS}$ & $* * * * *$ & $* *$ & $* * *$ \\
\hline Cinaz, 2004 [35] & CS & $* * * *$ & $* *$ & $* * *$ \\
\hline Dabbaghmanesh, 2007 [36] & CS & $* * * * * *$ & $*$ & $* * *$ \\
\hline Erdogan, 2001 [37] & CS & $* * *$ & $* *$ & $* * *$ \\
\hline Gashu, 2016 [38] & CS & $* * * * *$ & $* *$ & $* * *$ \\
\hline Giray, 2001 [39] & CS & $* * *$ & $* *$ & $* * *$ \\
\hline Hashemipour, 2008 [40] & CS & $* * * *$ & $* *$ & $* * *$ \\
\hline Keshteli, 2009 [41] & CS & $* * * *$ & $* *$ & $* * *$ \\
\hline Nourbakhsh, 2015 [42] & $\mathrm{CC}$ & $* * *$ & $*$ & $* *$ \\
\hline Ozenc, 2015 [43] & $\mathrm{CC}$ & & $*$ & $* *$ \\
\hline Rostami, 2018 [44] & $\mathrm{CC}$ & $* * * *$ & $*$ & $* *$ \\
\hline Salmonowicz, 2014 [45] & $\mathrm{CC}$ & $* * *$ & * & $* *$ \\
\hline Vanderpas, 1990 [46] & $\mathrm{CC}$ & $*$ & $*$ & $* *$ \\
\hline
\end{tabular}

$\mathrm{CS}=$ cross-sectional; $\mathrm{CC}=$ case-control

Note: A study can be awarded a maximum of four stars for case-controls and five stars for cross-sectional study within the selection category. A maximum of two stars can be given for comparability and three stars for outcome/exposure categories.

\section{Discussion}

Although discovered more than two centuries ago, Se and its role in health and disease remained underestimated and not fully elucidated. Endemic deficiency in large areas of central Asia clearly demonstrated its critical importance for survival. There are two well established entities of selenopenia: Kashin-Beck osteochondropathy and Keshan disease, a severe cardiomyopathy characterized by fulminant heart failure [47, 48]. The later mainly affects young children and women in childbearing age and it is apparent in population with particularly low Se intake $(<15 \mu \mathrm{g} / \mathrm{d})$, in some areas of China. It is noteworthy to mention that there is a relatively narrow range of Se intake, between deficiency $(<30 \mu \mathrm{g} / \mathrm{d})$ and toxicity $(>900 \mu \mathrm{g} / \mathrm{d})$ [49]. Numerous beneficial effects are attributed to Se and are extensively investigated in adults: antioxidant effects, correct functioning of the immune system, antiviral effects (nowadays of special interest), decrease in the risk of miscarriage, effects on bone metabolism, regulation of normal spermatozoa development and motility, beneficial effect on mood $[6,47,50,51]$. Interventional studies with Se in different pathological conditions in pediatric age are very rare.

In the current review, we selected 13 articles with the aim to investigate interdependence of Se levels and clinical presentation of endocrine diseases in children and adolescents. Majority of them refers to thyroid disorders and thyromegaly. Only two were performed in children with T1DM. It is expected finding taking into account that thyroid represents the organ which is most abundant in Se per gram of tissue, even richer than the brain, due to a high content of selenoproteins [6]. According to these data, the most frequent and severe Se deficiency is present in some parts of Africa (Northern Zair and Ethiopia) and Asia (China, Iran and Turkey) $[38,46,49]$. In a cross-sectional study on 628 children from Gonder, town of the Amhara region of Ethiopia, a goiter was found in $44,6 \%$ of them. The majority of children $(88,6 \%)$ had suboptimal iodine supply $(<100 \mu \mathrm{g} / \mathrm{l})$. The presence of Se deficiency (serum Se
$<70 \mu \mathrm{g} / \mathrm{l}$ ) was recognized as a problem in $57.8 \%$ of children [38]. Also, serum Se was negatively associated with T4 level in young children from this region. Vanderpas et al. emphasized that combined iodine and Se deficiency could be associated with the elevated frequency of myxedematous cretinism as a consequence of thyroid involution [46]. Similarly to observations from China, the distribution of Se deficiency was highly variable such that the deficiency and toxicity occurred in populations living just about $20 \mathrm{~km}$ apart, depending strongly on the geochemical characteristics of the soil in these specific areas $[46,49]$.

As mentioned above, in some severely iodinedeficient areas a concomitant deficiency of Se aggravates hypothyroidism leading to the myxedematous cretinism $[14,46]$. On the other hand, an adequate selenium supply protects the thyroid from damage due to excessive iodine exposure, thus a selenium deficit should be corrected before the introduction of iodine supplementation when both deficits coexist [14, 52, 53]. Selenium deficiency was negatively associated with serum T4, but almost all children had normal level of T3. Vanderpas et al. suggested that in iodine-deficient subjects, Se deficiency may help maintaining the T3 concentration at normal level [46]. We find that it is just opposite from what could be expected, taking into account the role of Se containing deiodinase DIO1 in conversion of T4 to T3. Improved peripheral deiodination of $\mathrm{T} 4$ should provide increase in T3/T4 ratio that is confirmed after Se supplementation in school children from Se deficient area, evident from the study of Contempre et al. [54]. From 11 studies concerning the interdependence of Se level and thyroid status, two demonstrated a positive correlation, one to T4 and another to T3. In the study of Gashu et al., Se concentration was negatively associated to $\mathrm{T} 4$ concentration, but positively to serum T3 concentration [38]. It is difficult to explain contradictory data about T4 and T3 levels, so further studies conducted on larger cohorts are necessary.

Endemic goiter is present in almost all regions of Turkey, while the highest prevalence is reported in the Black Sea region, reaching over $50 \%$, particularly in 
some high-altitude mountain villages. Iodization of salt was introduced in Turkey in 1968, but IDD (Iodine Deficiency Disorders) are far from eradication in this country [39]. For comparison, iodization of salt in former Yugoslavia started in 1956 and then reevaluated and reinforced in 1993, when the amount of iodine was doubled [55]. Giray et al. evaluated Se concentrations in goitrous children with severely and moderately iodine deficiency in comparison with normal and mildly iodine deficient non goitrous children serving as control, and found significantly lower Se values in the goitrous group. The same study showed lower level of antioxidant blood enzymes glutathione peroxidase, catalase and superoxide dismutase, suggesting alterations in antioxidant defense system and multiple micronutrient deficiency [39]. All Turkish studies reported high goiter persistence, despite normalization of the iodine status. Goitrous children from Turkey had moderate to severe Se deficiency in iodine depleted as well as in the iodine repleted areas [34, 39]. Similar to Turkish experience, the Iranian studies in former endemic areas showed high percentage of goiter in school children. The deficiency of both trace elements, iodine and selenium is even more severe than what was reported in Turkey. The most affected endocrine gland by Se deficiency was thyroid. Studies from Turkey, as well as from Iran referred goiter in children as the most prominent clinical sign of selenopenia (9 out of 11 studies). Unfortunately, even in the cross-sectional studies, Se level was not measured in the whole group, but only in goitrous children, selected by palpation of the gland when some severely Se deficient children might be omitted. In selected children with thyromegaly the widest difference in Se level between goitrous and non-goitrous children was found in the study of Aydin et al., $25.71 \mu \mathrm{g} / \mathrm{L}$ and $47.76 \mu \mathrm{g} / \mathrm{L}$, respectively [34]. The authors found significant negative correlation between thyroid volume and Se levels. On the other side, Gashu [38] and Hashemipour [40] did not find significant difference in Se levels between goitrous and non-goitrous children. Obviously, the role of other goitrogens in food or another traceelement deficiency should be additionally evaluated.

Extremely low Se level was found by Rostami et al. in the group of hypothyroid children [44]. Only one pediatric study from Iran, among selected in our review, referred to autoimmune thyroid disease. It failed to find significant difference in Se levels between patients with Hashimoto's thyroiditis and normal controls [42]. It is expected that having a normal Se level provides effective thyroid function and diminishes oxidative stress in thyroid cells, thus protecting thyroid from autoimmunity. In adult patients with chronic autoimmune thyroiditis from Germany and Greece, a Se supplementation decreased thyroid peroxidase antibodies [56, 57]. A striking majority of these patients reported an improvement in mood and well-being after 6 months of combined treatment (LT4+Se) [57]. In children, this effect was not confirmed [42]. However, a recent meta-analysis did not present sufficient data on clinical efficacy of selenium supplementation in chronic autoimmune thyroiditis in adults, and the authors concluded that further investigations are warranted [51].

Only two studies on children with T1DM were included as eligible in our systematic review [43, 45]. Hyperglycemia in diabetes increases or potentiates the oxidative stress. In the Turkish study a significantly lower levels of Se were found in diabetic children in comparison with controls and it correlated negatively with the HbA1c. Expectantly, the level of Se-containing enzyme, GPx was significantly higher in diabetic children [43]. We may explain this finding by enhanced the need for GPx antioxidant activity that consumes more Se in diabetic subjects. In the study of Polish authors, on a larger group of patients, difference in the GPx activity between diabetic children and non-diabetic controls was not confirmed [45]. It would be of interest to determine and to compare Se levels in different stages of diabetes in children (prediabetes, at admission, in acute metabolic decompensation, remission period, permanent stable or brittle diabetes). These results could be of immense practical importance for the prevention strategies.

\section{Strengths and Limitations}

To the best of our knowledge, this is the first review about the selenium deficiency in endocrine diseases to focus specifically on pediatric population, and thus has several strengths. First, we used a comprehensive methodological approach and careful selection of the studies, and also did an extensive data extraction from the included studies and provided a synthesis of evidence on this issue. Second, our search covered wide time interval and several different populations of children across different regions and countries. Further, the methodological quality of included studies was of moderate to high level, which was confirmed by a critical evaluation of their study design using appropriate evaluation scale.

However, there are some limitations that should be considered when interpreting our results. We only searched for published studies in English, Italian and Serbian language, thus we cannot exclude the possibility of publication bias and also, this might have affected the geographical representation of the studies. We did not limit our search to any particular endocrine disease since we wanted to present the general overview of the Se levels in endocrine disorders in children, available so far. Observational nature of the included studies is another limitation that should be acknowledged. Further randomized controlled trials are needed to adequately explore the role of Se in disorders of the endocrine system.

We were unable to perform a quantitative pooling of the data through a meta-analysis due to the lack of effect estimates in the included studies. Additionally, most of the included studies investigated populations from low and middle income countries thus our findings might not be widely applicable to other populations, particularly those from high-income countries, due to the pos- 
sible difference in life-style, habits and particularly in diet. In fact, lack of information on dietary habits and Se intake from food as well as the genetic background of the participants might have influenced our findings, but these were not available to us since they were not explored in the included studies. This further underlines the need for more research in that direction. In particular, since the incidence of endocrine diseases, especially diabetes is rising across the world, a trans-regional multicenter studies on children to explore Se levels across different populations and regions are highly desirable.

\section{Conclusions}

We may conclude that thyroid disorders, goiter and thyroid dysfunction are prominent signs of Se deficiency in school children. Critical importance of thyroid function on early brain development, somatic growth and maturation, as well as for appropriate energy level for an active life, is impetus for further thorough investigation of selenostasis in wide population, especially in children and adolescents. For thyroid autoimmunity, there are insufficient data in the pediatric age. Although deficiency of iodine and selenium are usually combined in some area, our systematic review showed that Se deficiency is

\section{References}

1. Margalioth EJ, Schenker JG, Chevion M. Copper and Zinc levels in normal and malignant tissues. Cancer 1983; 52:868-872. doi: 10. 1002/1097-0142(19830901)52:5<868::AID-CNCR2820520521>3.0. $\mathrm{CO} ; 2-\mathrm{K}$

2. Köhrle J, Jakob F, Contempré B, Dumont JE. Selenium, the thyroid, and the endocrine system. Endocr Rev 2005; 26:944-84. doi: 10.1210/er.2001-0034

3. Hincal F. Trace elements in growth: Iodine and selenium status of Turkish children. J Trace Elem Med Biol 2007; 21:40-43. doi: 10.1016/j.jtemb.2007.09.012

4. Mickiewicz B, Villemaire ML, Sandercock LE, Jirik FR, Vogel HJ. Metabolic changes associated with selenium deficiency in mice. BioMetals 2014; 27:1137-1147. doi:10.1007/s10534-014-9774-z

5. Jonklaas J, Danielsen M, Wang H. A pilot study of serum selenium, Vitamin D, and thyrotropin concentrations in patients with thyroid cancer. Thyroid 2013; 23:1079-1086. doi: 10.1089/thy.2012.0548

6. Duntas LH, Benvenga S. Selenium: an element for life. Endocrine 2015; 48:756-775. doi: 10.1007/s12020-014-0477-6

7. Toulis KA, Anastasilakis AD, Tzellos TG, Goulis DG, Kouvelas D. Selenium supplementation in the treatment of Hashimoto's thyroiditis: A systematic review and a meta-analysis. Thyroid 2010; 20:1163-1173. doi: 10.1089/thy.2009.0351

8. Beckett GJ, Arthur JR. 3 The iodothyronine deiodinases and 5'deiodination. Baillieres Clin Endocrinol Metab 1994; 8:285-304. doi: 10.1016/S0950-351X(05)80253-8

9. Arthur JR, Beckett GJ, Mitchell JH. The interactions between selenium and iodine deficiencies in man and animals. Nutr Res Rev 1999; 12:55-73. doi: 10.1079/095442299108728910

10. Arthur JR, McKenzie RC, Beckett GJ. Selenium in the immune system. J Nutr 2003; 133:1457-1459. doi: 10.1093/jn/133.5.1457s

11. Rayman MP. The importance of selenium to human health. Lancet 2000; 356(9225):233-241 doi: 10.1016/S0140-6736(00)02490-9

12. Kobayashi Y, Ogra Y, Ishiwata K, Takayama H, Aimi N, Suzuki KT. Selenosugars are key and urinary metabolites for selenium excretion within the required to low-toxic range. Proc Natl Acad Sci U S A 2002; 99:15932-15936. doi: 10.1073/pnas.252610699 important goitrogenic factor in school children. Deficiency of other trace elements, like $\mathrm{Zn}$ (cofactor of TSH receptor) and iron (cofactor of thyroid peroxidase enzyme), necessary for appropriate thyroid function, should be further investigated.

The role of Se in autoimmunity is attractive and promising, but it is underinvestigated and its clinical use is not recommended routinely. Some selenopenic subjects with autoimmune diseases could have beneficial effects. Wider use of Se is not advised because of its potential toxic effects. High Se intake may lead to selenosis or increase risk of type 2 diabetes mellitus, glaucoma, cancer, cardiovascular mortality and peripheral arterial disease [6]. Thus, it is particularly important to ensure optimal and balanced diet, in both macro and micronutrients, for better metabolic health from an early age.

Acknowledgments. This work was supported by grants from the Ministry of Education, Science and Technological Development of Republic of Serbia No. 31060, No. 41018, and Internal Project No. 37 of the Faculty of Medicine Nis, University of Niš, Serbia and was scientifically supported by the Association of Preventive Pediatrics of Serbia (Udruženje za preventivnu pedijatriju Srbije) 18000 Niš - Mediana, Blvd Zorana Djindjica 48, Clinical Centre Nis, Serbia.

13. Athanasopoulou H, Doulgeraki A. The importance of selenium in pediatrics. Trace Elem Electrolytes 2013; 30:100-104. doi: 10.5414/TEX01286

14. Zimmermann MB, Köhrle J. The impact of iron and selenium deficiencies on iodine and thyroid metabolism: Biochemistry and relevance to public health. Thyroid 2002; 12:867-878. doi: 10.1089/105072502761016494

15. Rayman MP. Selenium and human health. Lancet.2012; 379 (9822):1256-1268. doi: 10.1016/S0140-6736(11)61452-9

16. Wu Q, Rayman MP, Lv H, Schomburg L, Cui B, Gao C, et al. Low population selenium status is associated with increased prevalence of thyroid disease. J Clin Endocrinol Metab 2015; 100:4037-4047. doi: 10.1210/jc.2015-2222

17. Effraimidis G, Wiersinga WM. Mechanisms in endocrinology: Autoimmune thyroid disease: Old and new players. Eur $\mathbf{J}$ Endocrinol 2014; 170:R241-52. doi:10.1530/EJE-14-0047

18. Köhrle J, Gärtner R. Selenium and thyroid. Best Pract Res Clin Endocrinol Metab 2009; 23(6):815-827. doi: 10.1016/j.beem.2009. 08.002

19. Cardoso BR, Ong TP, Jacob-Filho W, Jaluul O, Freitas MIDÁ, Cozzolino SMF. Nutritional status of selenium in Alzheimer's disease patients. Br J Nutr 2010; 103:803-806. doi: 10.1017/ S0007114509992832

20. Thomson CD, Campbell JM, Miller J, Skeaff SA, Livingstone V. Selenium and iodine supplementation: Effect on thyroid function of older New Zealanders. Am J Clin Nutr 2009; 90 1038-1046. doi: 10.3945/ajen.2009.28190

21. Chanoine JP. Selenium and thyroid function in infants, children and adolescents. BioFactors 2003; 18:137-143. doi: 10.1002/biof. 5520190306

22. De Luca F, Santucci S, Corica D, Pitrolo E, Romeo M, Aversa T. Hashimoto's thyroiditis in childhood: presentation modes and evolution over time. Ital J Pediatr 2013; 39:8. doi: 10.1186/18247288-39-8

23. Vanderpump MPJ. The epidemiology of thyroid disease. Br Med Bull 2011; 99:39-51. doi:10.1093/bmb/ldr030 
24. Kishosha PA, Galukande M, Gakwaya AM. Selenium Deficiency a Factor in Endemic Goiter Persistence in Sub-Saharan Africa. World J Surg 2011; 35:1540-1545. doi: 10.1007/s00268-011-1096-5

25. Maritim AC, Sanders RA, Watkins JB. Diabetes, oxidative stress, and antioxidants: A review. J Biochem Mol Toxicol 2003; 17: 24-38. doi: 10.1002/jbt.10058

26. Hincal F, Basaran N, Celiker A, Bilgic A, Ozbarlas N, Baysal M. Selenium levels of children with dilated cardiomyopathy in Turkey. In: Abdulla M, Vohora S, Athar M, editors. Trace and toxic elements in nutrition and health. New Delhi: Wiley Eastern; 1995. pp. 175-8.

27. Yetgin S, Hincal F, Baçaran N, Ciliv G. Serum Selenium Status in Children with Iron Deficiency Anemia. Acta Haematol 1992; 88:185-188. doi: 10.1159/000204683

28. Burjonrappa SC, Miller M. Role of trace elements in parenteral nutrition support of the surgical neonate. J Pediat Surg 2012; 47: 760-771. doi: 10.1016/j.jpedsurg.2012.01.015

29. Senkevich OA, Koval'skiy YG. The Importance of Selenium in Children's Health and Reproduction. Importance of Selenium in the Environment and Human Health. IntechOpen 2020. doi:10.5772/ intechopen.88864

30. Moher D, Liberati A, Tetzlaff J, Altman DG. Preferred reporting items for systematic reviews and meta-analyses: the PRISMA statement. BMJ 2009; 339:b2535-b2535. doi:10.1136/bmj.b2535

31. The Newcastle-Ottawa Scale Coding Manual. Available: www.ohri. ca/\%0Aprograms/clinical_epidemiology/nos_manual.pdf

32. Herzog R, Álvarez-Pasquin MJ, Díaz C, Del Barrio JL, Estrada JM, Gil Á. Are healthcare workers intentions to vaccinate related to their knowledge, beliefs and attitudes? A systematic review. BMC Public Health. 2013; 13:154. doi:10.1186/1471-2458-13-154

33. Wells G, Shea B, O’Connell D. The Newcastle-Ottawa Scale (NOS) for assessing the quality of nonrandomised studies in meta-analyses. 2000 [cited 2 Jan 2021]. Available: http://www.ohri.ca/programs/ clinical_epidemiology/oxford.asp

34. Aydin K, Kendirci M, Kurtoğlu S, Karaküçük E, Kiriș A. Iodine and selenium deficiency in school-children in an endemic goiter area in Turkey. J Pediatr Endocrinol Metab 2002; 15:1027-1031.

35. Cinaz P, Karakasü DS, Çamurdan MO, Bideci A, Ayvalı ED, Yücel C. Goiter Prevalence, Serum Selenium, and Urine Iodine Status in a Previously Iodine-Deficient Area in Turkey. Biol Trace Elem Res 2004; 100:185-194. doi: 10.1385/BTER:100:3:185

36. Dabbaghmanesh MH, Sadegholvaad A, Ejtehadi F, Omrani G. Low serum selenium concentration as a possible factor for persistent goiter in Iranian school children. BioFactors 2007; 29:77-82. doi :10.1002/biof.552029207

37. Erdogan G, Erdogan MF, Sav H, Güllü S, Kamel N. Endemic Goiter, Thiocyanate Overload, and Selenium Status in School-Age Children. Biol Trace Elem Res 2001; 79:121-130. doi: 10.1385/BTER:79:2:121

38. Gashu D, Stoecker BJ, Adish A, Haki GD, Bougma K, Aboud FE, et al. Association of serum selenium with thyroxin in severely iodine-deficient young children from the Amhara region of Ethiopia. Eur J Clin Nutr 2016; 70:929-934. doi: 10.1038/ejcn.2016.27

39. Giray B, Hincal F, Teziç T, Ökten A, Gedik Y. Status of Selenium and Antioxidant Enzymes of Goitrous Children Is Lower Than Healthy Controls and Nongoitrous Children with High Iodine Deficiency. Biol Trace Elem Res 2001; 82:35-52. doi: 10.1385/ BTER:82:1-3:035

40. Hashemipour M, Siavash M, Amini M, Aminorroaya A, Rezvanian H, Kachuei A, et al. Goiter Persistence After Iodine Replenishment, the Potential Role of Selenium Deficiency in Goitrous Schoolchildren of Semirom, Iran. Exp Clin Endocrinol Diabetes 2007; 116:75-79. doi: 10.1055/s-2007-990298

41. Keshteli AH, Hashemipour M, Siavash M, Amini M. Selenium Deficiency as a Possible Contributor of Goiter in Schoolchildren of Isfahan, Iran. Biol Trace Elem Res 2009; 129:70-77. doi: 10.1007/ s12011-008-8296-3
42. Nourbakhsh M, Ahmadpour F, Chahardoli B, Malekpour-Dehkordi Z, Nourbakhsh M, Hosseini-Fard SR, et al. Selenium and its relationship with selenoprotein $\mathrm{P}$ and glutathione peroxidase in children and adolescents with Hashimoto's thyroiditis and hypothyroidism. J Trace Elem Med Biol 2016; 34:10-14. doi: 10.1016/j.jtemb.2015.10.003

43. Özenç S, Saldir M, Sarı E, Çetinkaya S, Yeşilkaya Ş, Babacan O, et al. Selenium, zinc, and copper levels and their relation with $\mathrm{HbA1c}$ status in children with type 1 diabetes mellitus. Int J Diabetes Dev Ctries 2015; 35:514-518. doi: 10.1007/s13410-015-0327-y

44. Rostami S, Fathollahpour A, Abdi M, Naderi K. Alteration in Prooxidant-Antioxidant Balance Associated with Selenium Concentration in Patients with Congenital Hypothyroidism. J Med Biochem 2018; 37:355-363. doi: 10.1515/jomb-2017-0052

45. Salmonowicz B, Krzystek-Korpacka M, Noczyńska A. Trace Elements, Magnesium, and the Efficacy of Antioxidant Systems in Children with Type 1 Diabetes Mellitus and in Their Siblings. Adv Clin Exp Med 2014; 23:259-268. doi: 10.17219/acem/37074

46. Vanderpas JB, Contempré B, Duale NL, Goossens W, Bebe N, Thorpe $\mathrm{R}$, et al. Iodine and selenium deficiency associated with cretinism in northern Zaire. Am J Clin Nutr 1990; 52:1087-1093. doi: 10.1093/ajcn/52.6.1087

47. Guastamacchia E, Giagulli V, Licchelli B, Triggiani V. Selenium and Iodine in Autoimmune Thyroiditis. Endocrine, Metab Immune Disord Targets 2015; 15:288-292. doi: 10.2174/ 1871530315666150619094242

48. Chanoine J-P, Nève J, Wu S, Vanderpas J, Bourdoux P. Selenium Decreases Thyroglobulin Concentrations But Does Not Affect the Increased Thyroxine-to-Triiodothyronine Ratio in Children with Congenital Hypothyroidism 1. J Clin Endocrinol Metab 2001; 86: 1160-1163. doi: 10.1210/jcem.86.3.7312

49. Ashton K, Hooper L, Harvey LJ, Hurst R, Casgrain A, FairweatherTait SJ. Methods of assessment of selenium status in humans: A systematic review. Am J Clin Nutr 2009: 89: 2025S-2039S. doi:10. 3945/ajcn.2009.27230F

50. Duntas LH. Selenium and the Thyroid: A Close-Knit Connection. J Clin Endocrinol Metab 2010; 95:5180-5188. doi: 10.1210/jc.20100191

51. Winther KH, Wichman JEM, Bonnema SJ, Hegedüs L. Insufficient documentation for clinical efficacy of selenium supplementation in chronic autoimmune thyroiditis, based on a systematic review and meta-analysis. Endocrine 2017; 55:376-385. doi: 10.1007/s12020016-1098-z

52. Bartalena I, Tanda M, Piantanida E, Lai A, Compri E, Lombardi V. Environment and thyroid autoimmunity. In: Wiersinga W, Drexhage H, Weetman A, editors. The Thyroid and Autoimmunity. Stuttgart: Thieme; 2007. pp. 60-73.

53. Saranac L, Zivanovic S, Bjelakovic B, Stamenkovic H, Novak M, Kamenov B. Why Is the Thyroid So Prone to Autoimmune Disease. Horm Res Paediatr 2011; 75:157-165. doi: 10.1159/000324442

54. Contempré B, Duale NL, Dumont JE, Ngo B, Diplock AT, Vanderpas J. Effect of selenium supplementation on thyroid hormone metabolism in an iodine and selenium deficient population. Clin Endocrinol (Oxf) 1992; 36:579-583. doi: 10.1111/j.1365-2265.1992. tb02268.x

55. Sinadinovic J, Han R. Iodine deficiency, endemic goiter and prophylaxis. Monography (In Serbian). Belgrade: Politop Belgrade Ed; 1995.

56. Gärtner R, Gasnier BCH, Dietrich JW, Krebs B, Angstwurm MWA Selenium Supplementation in Patients with Autoimmune Thyroiditis Decreases Thyroid Peroxidase Antibodies Concentrations. J Clin Endocrinol Metab 2002; 87:1687-1691. doi: 10.1210/jcem.87.4.8421

57. Duntas L, Mantzou E, Koutras D. Effects of a six month treatment with selenomethionine in patients with autoimmune thyroiditis. Eur J Endocrinol 2003; 148:389-393. doi: 10.1530/ eje.0.1480389 